\title{
An enhancement to ACT: A stand-alone disk driver compatible with RDOS
}

\author{
JOHN G. MARRIOTT and RONALD E. VOIGTMAN \\ Parke, Davis and Company Research Laboratories, 2800 Plymouth Road, Ann Arbor, Michigan 48106
}

\begin{abstract}
An extension to the ACT computer language was described which provides for the storing of data on a high-speed moving head disk. Data stored on disk may then be retrieved and analyzed using the disk-based operating systems and high-level computer languages, such as FORTRAN and BASIC, available from the computer manufacturer. Advantages as well as limitations of the extended version of ACT were discussed.
\end{abstract}

Laboratory automation poses a dilemma. On the one hand, it promises to bring relief from the routine and time-consuming tasks that accompany almost any type of data collection and analysis. On the other hand, the time and effort involved in developing and implementing the new technology often threatens to deter the pursuit of the very knowledge it is intended to advance. Thus, while it may be a very desirable or even necessary goal, automating a laboratory entails research costs beyond the initial price of the equipment and its installation.

One answer to this dilemma for the behavioral laboratory is the availability of several "turn-key" systems such as Interact (BRS/LVE) and Grason-Stadler's SCAT (see Wood, Sette, \& Weiss, $19^{-7} 5$ for a recent review of these systems). These are conplete systems consisting of a general-purpose minicomputer, digital interface, and supporting software. The interfaces are designed to attach directly to various test environments commonly found in the psychology laboratory. Simple, high-level programming languages and operating systems, that can be mastered with a modest investment in time and effort (a few hours), relieve the experimenter of the necessity of learning a complex assembly or machine language unique to the particular hardware purchased. Thus, packages such as Interact, SCAT 3002, and PDP-8/SKED allowed the researcher to bring the digital computer into the laboratory to collect and analyze data with a minimum of set-up and development time, yet still provide the advantages of computer automation.

In reality, even the systems mentioned above

We gratefully acknowledge the cooperation of the BRS/LVE division of Tech Serv, Inc., in providing the information necessary to implement the changes to ACT described herein. Tom Allen of the Data General Corporation, Detroit Office, did most of the programming described. His assistance is greatly appreciated. Reprints of this article and listings of the software described are available from the authors. Address requests to: John G. Marriott, Parke-Davis Research Laboratories, 2800 Plymouth Road, Ann Arbor, Michigan 48106. impose limitations on the type of experimental procedure that can be handled without recourse to additional equipment or software development. Although they are well suited to the process-control functions of the more conventional behavioral testing procedures. e.g., operant conditioning, they are often inadequate to perform more sophisticated or complex testing procedures. Experiments requiring either large numbers of inputs or outputs, for example, or the processing of analog information would call for additional hardware. Software limitations become apparent when the hardware is modified to accommodate special needs. Furthermore, these systems often do not support computer peripherals that a researcher may find vital for his application or may not provide an adequate number of variables, constants, or timing facilities for complicated application programs.

The most serious deficiency encountered in the use of these systems, however, is that they are primarily designed to handle only half of the typical experimental procedure: the data-collection or process-control portion. The other half-concerned with data reduction and analysis-must be handled by systems and software developed by the user or acquired from other sources. Few researchers, having once acquired a general-purpose minicomputer, would be content to have it function solely as a controller or data collector, leaving to other resources the data-analysis functions. Rather, they would prefer to utilize the same system either alternately or simultaneously, to perform control, collection, and analysis functions. This is certainly within the scope of the systems currently available. Therefore, while they represent an advance over the do-it-yourself approach, many will find these systems alone do not meet normal expectations for an automated data-collection and analysis facility.

A partial solution to some of these difficulties, as suggested by Wood et al. (1975). would be to merge the process-control language and interface available from the behavioral equipment vendors with the 
operating systems and software (RDOS, FORTRAN, BASIC) available from the computer manufacturer. Under this scheme, the data-collection functions could be carried out by the special-purpose interface, using a simple process-control programming language and operating system, while the data analysis and computing functions are performed by the sophisticated languages and operating systems available from the computer manufacturer.

This report describes an attempt to merge the ACT (Automated Contingency Translator) programming language (Millenson, 1971) and Interact control system with the Real-Time Disk Operating System (RDOS) of the Data General Corporation. The system was designed specifically to: (1) add to the ACT operating system the capability of storing information on a high-speed moving-head disk; (2) create disk files using RDOS for the storage of data collected using Interact; (3) communicate between RDOS and the ACT operating system the information necessary for writing into the appropriate disk files. These three functions are accomplished by three programs described below. All hardware and software necessary to add the disk and disk operating system to the Interact system are available from the Data General Corporation.

\section{PROGRAM DESCRIPTIONS}

The first program required is a stand-alone disk driver for use with the ACT operating system. This assembly language routine writes a $400_{8}$ block of core memory onto the rotating disk memory. The data buffer already available in ACT III $^{1}$ was enlarged to the appropriate size and serves as the core area written onto disk. All output can be directed to the data buffer by selecting the appropriate sense switch on the computer console and then striking "Ctrl D" on the control terminal. Data collected in the buffer can then be dumped onto disk by: (1) the data buffer becoming full; (2) keyboard command from the control console ( $\mathrm{Ctrl} \mathrm{T}$ ); (3) TAPE command in the state declaration line of any application program which is executed when that state is entered. Data are collected on the disk just as they would be transmitted to the output terminal. Within the disk file, the data are written sequentially just as they were collected, with no sorting of the data into user files at this point.

The disk file which receives the data collected by ACT is created by a FORTRAN program which runs under RDOS. Since the disk drive routine simply increments a disk address each time a record is written. it requires that the data file have a contiguous structure (rather than a random or sequential structure). Such a file structure limits the complexity of the disk drive routine. After calling the program which creates the contiguo: 1 s file, the operater must enter the name and size of the file to be created. The name may be any RDOS-compatible character string and the size is limited only by the amount of contiguous space available on the disk. If the name does not conflict with others in the directory, and space is available, the program returns the starting address and octal size of the file. This information must be entered into the ACT operating system using the third program.

Files containing data from previous experiments may be reinitialized, cleared of their contents, and made available for further data collection, using this same pregram. By simply indicating to the program that the file already exists (by responding "OLD"), the same data file may be used day after day. Using this feature, data could be sorted into individual user files each day and analyzed by programs such as those described by others at this conference (Flannigan, 1976). The original data file may then be cleared and readied for new data collection.

The final piece of software is a short assembly-language routine that communicates the starting address and size of the output file to the ACT operating system. Error checking, to prevent inadvertent destruction of data already on disk. also occurs at this point. This program is loaded into the computer along with ACT. Prior to running ACT, the disk file information is entered via the sense switches on the computer console using this program.

Once the disk information is entered, the operation of ACT proceeds as usual. Should the amount of data exceed the space allotted on disk, no further data collection on disk will occur. Output to other devices will continue as usual. Disk errors will halt the system. A desirable future modification would be to return error codes to the control terminal, such as the E-1 through E-4 messages currently available in ACT, rather than to halt the system on a disk error.

When the data-collection phase is complete, the operator may switch from the ACT operating system back to RDOS. This is a quick and simple procedure requiring just a few seconds using the auto-load option and bootstrap located on disk. Once RDOS is operational. the data sorting. summarizing, and analyzing may proceed.

\section{ADVANTAGES}

The major advantage offered by this revised version of ACT is the compatibility it provides between the data-collecting capabilities of Interact and the dataanalyzing capabilities of RDOS and its accompanying languages. Because of the common disk medium, it is no longer necessary to reenter data into the computer or to sort data tapes manually in order to complete an experimental analysis using the computer. Rather, the 
data can be manipulated automatically by the machine. As soon as an experiment is complete, all that is necessary in order to analyze the test results is to change from one operating system to the other and initiate the new programs. Data collected with ACT can then be retrieved, sorted, and analyzed automatically by programs running under RDOS.

Since data collected by ACT may be volatile, e.g., the value of a constantly changing variable at particular times during an experiment, the speed and efficiency of the data buffer and disk storage has the added value of minimizing the loss or erroneous recording of sensitive data. In this sense, the use of the disk with ACT resembles the operation of an almost inexhaustible storage medium which closely rivals the speed and reliability of core memory.

The most apparent improvement in daily operations to those accustomed to the use of paper tape is the speed and convenience with which routine operations can be performed using the disk. Loading programs and switching from one operating system to another can be accomplished in a matter of seconds. Numerous other attractive features, such as editors, extended compilers, and debugging routines that can be loaded under key. board command, are available under the disk operating system.

\section{DISADVANTAGES AND LIMITATIONS}

A serious drawback involved in adding a disk to the Interact system is the initial cost of the disk drive and controller. The addition of a single disk cartridge drive can double the price of a small InterACT system. Not only the disk hardware, but also additional core memory must be purchased (24K words minimum) in order to support RDOS and FORTRAN IV. Since they are primarily mechanical devices, rather than electronic, the price of disk drives has not diminished in recent years as dramatically as those of computers in general. Nor is it likely that they will decline much in the future. The amount of storage available on a single drive, however, has increased, while drive costs have remained steady.
Thus, while it is of little importance to the small user, costs per byte stored have decreased. Nonetheless, the disk itself represents a sizable portion of the total hardware investment for an Interact system with disk. Needless to say, the addition of the disk represents a sizable gain in the quality of the system and its capabilities.

The programs added to the ACT operating system described above require approximately $400_{8}$ core locations that might otherwise be used for data storage or user programs. This brings the amount of core required for the modified ACT operating system to near $4 \mathrm{~K}$ words. This is still a modest amount of the $24 \mathrm{~K}$ word machine required by RDOS and should not effect the operation of most systems.

The most serious limitation is that the enhancements do not constitute in any sense a disk operating system for ACT. Ideally, ACT could be used to create files for each user, sort data, load programs from disk, and analyze data, simultaneous with data collection and process control functions. None of these features are made possible by the system described. It is hoped that this first step to utilize a disk with ACT will encourage others to make additional improvements that will someday result in an easy-to-use, yet powerful, real-time data acquisition and analysis system.

\section{REFERENCES}

Flannigan, K. Data nianıpulation and reduction in a $32 \mathrm{~K}$ environment. Behavior Research Methods \& Instrumentation. 1976. 8, 247.248.

Millenson. J. R. A programming language for on-iine control of psichological experiments. Behavioral Science. $19^{7} 1$. 16. $248-256$

WoOd R. W. Serte. W. F.. \& Weiss, B. Intertacing the expermenter to the computer. Languages for psychologists. Amertcun Psychoiogest, 19-5, 30, 230.238.

\section{NOTE}

1. A complete description of the InterACT system and ACT language are found in The ACT Primer and The InterACT Technical Manual available from BRS/LVE division of Tech Serv, Inc. 